\title{
The effect of Kolkolis in reducing Blood pressure
}

\author{
Dennis Glen Ramos*, Sheena Vie Alimboyao, Alhazel Dianne Dompiles, Emerald Gem Nazarro, Nicole Mathews Pascua, Kristine Joy \\ Pawid, Zandrex Reyes and Albert Vera \\ RNMSN, Faculty, School of Nursing, St Louis University, USA
}

\begin{abstract}
Hypertension is a common health risk afflicting more than 1 billion people worldwide. Complementary Alternative Medicines (CAM), such as Massage and Reflexology, have been used alongside medical treatment to control Hypertension. Presently, many studies found that massage and reflexology therapy both adversely affect the blood pressure. Kolkolis is an indigenous foot massage in Cordillera that utilizes bamboo or pine sticks to massage the calves and feet. The effects of Kolkolis as a therapeutic intervention used in clinical practice have not been abundantly explored, so the study intends to find the effect of Kolkolis in reducing blood pressure among hypertensive clients. It also sought to find if there is a significant difference in the effect of Kolkolis in the reduction of blood pressure along duration. The result revealed that Kolkolis is an effective adjunct therapeutic intervention that can be used to reduce blood pressure. Furthermore, duration is an intervening factor in blood pressure reduction.
\end{abstract}

\section{Introduction}

The modern era has undergone a rapid heath revolution governed by swift urbanization and the globalization of unhealthy lifestyles such as sedentary lifestyles, overdependence on medications, chronic smoking and drinking. The most striking shift of these changes that can be seen is the increasing number of non-communicable diseases, such as cardiovascular disease, hypertension, diabetes, and cancer, afflicting all kinds of people throughout the world (World Health Organization, 2013) [1]. Hypertension or high blood pressure is a common health risk that can lead to heart disease, stroke, disability and death [2]. The Seventh Report of the Joint National Committee on Prevention, Detection, Evaluation and Treatment of High Blood Pressure. Chobanian [3], defined Hypertension as having a systolic blood pressure greater than $140 \mathrm{mmHg}$ and a diastolic pressure greater than $90 \mathrm{mmHg}$ based on the average of two or more accurate blood pressure measurements taken during two or more contacts with a health care provider.

Hypertension remains to be one of the top leading causes of morbidity in the world causing more than 7.5 million deaths and affecting 1 billion more people. Presently, about 14 Million Filipino adults are Hypertensive according to the Food and Nutrition Research Institute of the Department of Science of Technology (FNRI - DOST). In the Cordilleras, it remains to be one of the top five leading causes of morbidity, afflicting about 19,000 people (Department of Health Cordillera Administrative Region, 2009). In Baguio City, approximately 2364 people are afflicted with Hypertension and one of the leading causes of morbidity affecting 703 people (Baguio Health Department Report, 2013).

Presently, Non-pharmacologic and alternative life-style based treatments have become a common part of holistic health care. The National Center for Complementary and Alternative Medicine (NCCAM) in the USA describes Complementary and Alternative Medicine (CAM) as a group of diverse medical and healthcare systems, practices, and products that are not presently considered as part of conventional medicine [4]. Massage is one of the commonly used CAM by individuals.

Massage therapy is the manipulation of superficial and deeper layers of muscle and connective tissue using various techniques in order to enhance function, aid in the healing process, decrease muscle reflex activity, inhibit motor-neuron excitability, and promote relaxation and well-being. On the other hand, Reflexology, or zone therapy, is a specialized category of massage involving the physical act of applying pressure to the feet, hands, or ears with specific thumb, finger, and hand techniques without the use of oil or lotion. Reflexology is a focused pressure technique based on the premise that there are zones and reflexes on different parts of the body which corresponds to and are relative to all parts, glands and organs of the entire body. McVicar et al. [5], found that reflexology was significant in reducing cardiac parameters such as blood pressure and pulse rate. Ultimately, experts believe that the effects of reflexology are immediate regardless of intervention, but it often depends on the person's perceived status.

One of the indigenous massages used in the Cordilleras is Kolkolis, a traditional bamboo reflexology massage administered for its' therapeutic benefits. Kolkolis is a form of foot therapy which utilizes pine sticks or bamboo to massage the calves and feet. It is said that people would be relieved of tension and stress by using the strength and durability of all-natural bamboo (Philippine Spa Massage Workshop, 2014) [6]. This can be aligned with Fitzgerald and Bowers'Zone Theory (1917) [7], the theory posits that the body is divided into ten vertical zones, with each zone corresponding to fingers and toes that extend from the head. In theory, every organ lies in a specific zone that can be accessed through a point or area on the feet or hands.

Correspondence to: Dennis Glen Ramos, RNMSN, Faculty, School of Nursing, St Louis University, USA, E-mail: denski74@yahoo.com

Keywords: effect, kolkolis, blood pressure

Received: June 18, 2015; Accepted: July 23, 2015; Published: July 26, 2015 
Presently, many studies found that massages and reflexology therapy both adversely affect the blood pressure. Many researchers believe that using massage can be viable in the maintenance of blood pressure and many ongoing researches continue to further expound on the effectively of this particular intervention. Recently, studies revealed that massage can effectively and independently decrease systolic blood pressure [8] and decrease diastolic blood pressure [9]. Likewise, studies have also revealed that massage was effective in decreasing both systolic and diastolic blood pressure $[10,11]$. This is further supported by several studies establishing that reflexology therapy can be used to significantly reduce both systolic and diastolic blood pressure, pain, and anxiety [12-14].

Akin to other studies exploring different types of massages, Kolkolis is a massage that is typically unknown to others outside the Cordillera Region of the Philippines. Consequently, the effects of Kolkolis as a therapeutic intervention used in clinical practice have not been abundantly explored, warranting a need to conduct this study.

Thus this study intended to find the effect of Kolkolis in reducing blood pressure among hypertensive clients. It also wished to find if there is a significant difference in the effect of Kolkolis in the reduction of blood pressure along duration.

\section{Method}

The study is a quasi- experimental research that utilized a crossover design. The locale and population includes residents of Baguio City diagnosed with Hypertension with co-morbidities limited to Diabetes Mellitus, Cardiovascular Disease and Kidney Diseases, have no severe integuementary disease and over 60 years old. Screening and selection of respondents was done using the inclusion criteria. A total of 64 respondents were included in the study. The respondents were randomly assigned into two groups using the fishbowl method with 32 respondents in each group. After the study, two respondents were considered as attrition as a result of withdrawal secondary to changed residencies. History taking and informed consent were done and contact numbers were taken for follow up. Biophysical measures specifically the Blood Pressure was used to interpret the reduction of BP.

An aneroid Sphygmomanometer was used in the study. All tools were inspected and recalibrated using a revised protocol set forth by the European Society of Hypertension International Protocol revision 2010 for the validation of blood pressure measuring devices in adults [15]. To ensure the accuracy of data gathering, each researcher had undergone competency retraining in acquiring blood pressure using the guidelines set forth by the National Institute of Health and Clinical Excellence (NICE, 2011) [16].

A consent form was given to those respondents who participated in the research, along with the premise that they may withdraw at any time without the use of coercion to join or continue to take part in this research. Stand by first aid kits were made available in case of a hypertensive crisis may occur. In case of any emergencies, the respondents will be endorsed under the care of Saint Louis University Hospital of the Sacred Heart under the care of Dr. Romulo Dumaguing. During the sessions, the researchers instructed every respondent to refrain from doing activities that may alter their blood pressure such as talking on the phone or to other respondents; they were also informed that all therapeutic session were to be held in the same place at the same time between 12-4 PM.

During the Kolkolis sessions, the respondents had their feet elevated at an appropriate level for the procedure as determined by the specialist and the leg was evenly supported to prevent compression of blood vessels. The specialist used two small sticks approximately 5 inches long wherein they were instructed to apply short strokes to the different pressure points of the feet, followed by a massage to the calves. The duration of therapy, the time spent on each foot and its respective party was dictated and instructed by the researchers. Pressure applied was also dictated by the amount of time endorsed to the kolkolis specialists.

During the first therapeutic session, group A were asked to sit in a comfortable chair wherein their initial blood pressures were taken twice. Group A received Kolkolis for 30 minutes with the following parameters: 15 minutes per foot with the following sub-sets of 1 minute massage to the calves, 4 minutes reflexotherapy to the arches of the foot, and 5 minutes to the soles and metatarsal regions of the foot. Sources of possible stressors were contained by the researchers. After 30 minutes, group A waited for 5 minutes before their Blood Pressure were taken twice. At the same time, group B were asked to sit in a comfortable chair with their initial blood pressure were taken twice. Group B received Kolkolis for 20 minutes with the following parameters: 10 minutes per foot with the following sub-sets of 1 minute massage to the calves, 2 minutes reflexotherapy to the arches of the foot, and 3.5 minutes to the soles and metatarsal regions of the foot. After 20 minutes of Kolkolis, Group B waited for 5 minutes before having the Blood pressure taken twice. Afterwards, both Groups were acknowledged as well the experts and instructed to return at the same place during the same time after a wash out period of 5 days.

During the second therapeutic session, group A received 20 minutes of Kolkolis, and Group B received the 30 minutes. The same procedure as the first Kolkolis session was done.

To determine the effect of Kolkolis in the reduction of blood pressure among hypertensive patients, frequency was used in the pretest - post-test results. To determine the significant effect of Kolkolis in the reduction of blood pressure among hypertensive client along duration, mean and $\mathrm{T}$ test was used.

\section{Results and discussions}

This chapter presents the analysis, interpretation of data and implications of the findings of the study regarding the effect of Kolkolis in reducing blood pressure.

Table 1 shows the effect of Kolkolis on systolic and diastolic blood pressure along duration. The table further revealed that the 20 minutes Kolkolis is partially effective in reducing systolic blood pressure with an average of $-14.596(\mathrm{PE})$ and diastolic blood pressure with an average of -5.56452 (PE). Likewise, it also reveals that Kolkolis is partially effective in reducing blood pressure along a 30 minute duration, with a decrease of systolic blood pressure averaging to -1.2903 (PE) and diastolic blood pressure averaging to 1.370968 (PE).

Table 1. Effect of Kolkolis on systolic and diastolic blood pressure along duration.

\begin{tabular}{|c|c|c|c|}
\hline Duration & $\begin{array}{c}\text { Systolic Blood } \\
\text { Pressure }\end{array}$ & $\begin{array}{c}\text { Diastolic Blood } \\
\text { Pressure }\end{array}$ & Interpretation \\
\hline 20 Minutes & -14.596 & -5.56452 & PE \\
\hline 30 Minutes & -1.2903 & 1.370968 & PE \\
\hline Systolic Blood Pressure Scale & Diastolic Blood Pressure Scale \\
\hline$(-80.00)-(-50.00)$ & HE & $(-35.00)-(-21.25)$ & HE \\
\hline$(-49.99)-(.20 .00)$ & E & $(-21.24)-(-7.5)$ & E \\
\hline$(-19.99)-(.10 .00)$ & PE & $(-7.4)-(6.25)$ & PE \\
\hline$(9.99)-(40.00)$ & NE & $(6.26)-(20.00)$ & NE \\
\hline
\end{tabular}


The reduction of blood pressure may due to a decrease in the stress and anxiety levels as Kolkolis allows them to feel relaxed and calm after the treatment thereby decreasing anxiety. Although stress and anxiety levels were not assessed during the study, this may be determined through biophysical measures such as blood pressure. Therefore, the clients' feeling of relaxation and comfort after the administration of Kolkolis may indirectly decrease blood pressure as an effect. Kuhn [17] hypothesized that the mechanism of reflexology is that when pressure is applied to the reflex points, it stimulates blood flow and nerve impulses thereby releasing chemicals such as endorphins and toxins, thus creating homeostasis in the body. Several research found that massage and reflexology therapy are effective in reducing stress [11], Anxiety [18-20], and vital signs [21,22]. They discussed that the mechanical stimulation of nerve receptors of the skin has a calming effect and emotional affect, thus decreasing effect of nerve activity from the massage.

Moreover, research suggests that massage and reflexology is effective in decreasing blood pressure, even with receiving twenty minutes of intervention. According to Guan [23], there is an increased PNS activity and decreased SNS activity by 20 minute single session of massage therapy. Sympathetic inhibition leads to a drop of peripheral resistance, while parasympathetic activation leads to a depressed heart rate (reflex bradycardia) and contractility.

Delaney et. al. [24], found that the use of trigger point massage found that a twenty minute massage was a feasible amount of time for a significant decrease in heart rate, systolic blood pressure, and diastolic blood pressure. Analysis found that there was a significant increase in the amount of parasympathetic activity following the triggerpoint massage. Black and Hawks [25] discussion of pressure applied therapies, therapies such as Kolkolis stimulates the pressure receptors in the feet that are connected to the dorsal horn of the spine, travelling through the brain and stimulating the parasympathetic nervous system (PNS). Stimulation of the PNS leads to a decrease in total peripheral resistance in blood vessels, causing dilation, improvement of blood flow and increasing oxygen saturation in the body, thereby decreasing blood pressure.

Regardless, it can still be inferred that Kolkolis is effective in reducing blood pressure. It can also be deduced that twenty minutes is a feasible enough of time for significant physiological changes. This shows that massages and reflexology therapies such as Kolkolis do affect the cardiovascular system. Furthermore, this is beneficial for the consumers as it becomes a complementary alternative medicine that is an efficient, cost-effective and less time consuming intervention to do.

Table 2 shows the difference in the effect of Kolkolis in reducing Blood Pressure along durations. The tabular $\mathrm{p}$ value of 0.05 is less than the computed value on systolic $\left(2.38 \times 10^{\wedge} 7\right)$ and diastolic $(4.77 \times$ $10^{\wedge} 7$ ). This shows that 30 minutes is more effective in reducing both systolic and diastolic blood pressure as compared to 20 minutes.

The significant difference in blood pressure along duration may be due to the longer period of pain fiber inhibition. According to the Gate Control Theory by Melzack and Wall [26] Kolkolis allows the respondents to experience more time being free of painful and stress

Table 2. Effect of Kolkolis on blood pressure reduction along duration.

\begin{tabular}{|l|l|l|}
\hline & $\mathbf{P}(\mathbf{T}<=\mathbf{t})$ Two-tail Value & Interpretation \\
\hline Systolic Blood Pressure & 0.0000000477188696289969 & Significant \\
\hline Diastolic Blood Pressure & 0.0000293718510528594 & Significant \\
\hline Df: 61 & $\mathrm{P}<\alpha 0.05$ & Interpretation: Reject Ho \\
\hline
\end{tabular}

aggravating stimuli. The impact of having a longer duration of time for foot massage allows for longer periods of diminished sensations of pain in their feet, feelings of relaxation, increased energy, and increased ability to manage work demands Jeong [27]. This can be supported by a research by Moeini [12] which found that with 30 minutes reflexotherapy, systolic blood pressure, diastolic blood pressure, heart rate and respiratory rate reveals decreased. Sherman et al. (2014) found that by comparing the effectivity of 30 minutes to 60 minutes therapeutic massage, the 30 minute treatments were not significantly better compared to 60 minute massages where there was a significant increase of improvement on neck dysfunction and pain intensity. According to Zone Theory, the different zones on the feet correspond to different parts and organs of the body. The manipulation of said zones of the feet can change the physiology of the human body. Thus a longer application of kolkolis to these particular zones may provide for a more pronounced change in physiology like blood pressure. When reflexotherapy is immediately followed by a massage, there is reduction in the tightness in the muscles, improved blood flow and breathing, plus reduced anxiety and stress. Therefore, it can be assumed that the longer the duration, the more effective the intervention in increasing range of motion, strengthen the immune system and provide an improved sense of well-being.

Another reason for the difference in blood pressure along duration could be due to the adherence to the Meridian cycle. Jeong [27], suggests that massages that last for 30 minutes follows the meridian cycle of 28 minutes wherein the baroreflex may maximize blood pressure reduction. This is supported by the study of Frankel [28] who explored the effects of the foot reflexology on the baroreceptor reflects which control blood pressure. The study found out that baroreceptor reflex sensitivity was significantly lowered in the intervention groups compared to the control groups. So, Kolkolis group has lower baroreceptor sensitivity thereby decreases blood pressure. Thus, it is suggested that 30 minutes massage could be more effective in reducing blood pressure.

\section{Conclusion}

The Study concludes that: Kolkolis is a cost effective alternative intervention in reducing blood pressure and duration of Kolkolis is an intervening factor in reducing blood pressure.

Majority of the population choose CAM in conjunction with other medication treatment because it is a financially feasible, natural, safe that can be used as an intervention in both rural and urban settings.

\section{Recommendation}

The researchers recommend the following: a) Encouraging senior citizens to utilize Kolkolis as an adjunct treatment alongside traditional pharmacologic intervention for the reduction of blood pressure, b) To conduct similar research with a larger population size and variations in population to include different age groups with hypertension. And c) Kolkolis can be offered by community nurses as an adjunct therapy alongside traditional interventions in lowering blood pressure among hypertensive clients. Nurses will have to conduct information education campaign to increase awareness of the community to utilize Kolkolis as one of the complementary alternative medicine. Nurses will train the people in the community such as the BHW's, elderlies and people without work on how to properly implement the skill.

\section{Reports}

Baguio Health Department Report 2013, Baguio Health 
Department, Baguio City Philippines Food and Nutrition Research Institute of the Department of Science of Technology (FNRI -DOST) Report (2010), Philippines.

\section{References}

1. World Health Organization. (2013, April 13). A Global Brief on Hypertension. Retrieved November 17, 2014.

2. France CR, Ditto B (1996) Risk for high blood pressure and decreased pain perception Current Directions in Psychological Science 5: 120-125.

3. Chobanian AV, Bakris GL, Black HR, Cushman WC, Green LA, et al. (2003) The Seventh Report of the Joint National Committee on Prevention, Detection, Evaluation, and Treatment of High Blood Pressure: the JNC 7 report. JAMA 289: 2560-2572. [Crossref]

4. National Center for Complementary and Alternative Medicine. Complementary, Alternative, or Integrative Health: What's In a Name? (2014, July 15). Retrieved November 25, 2014

5. Mc Vicar A, Greenwood C, Fewell F, D’Arcy V, Chandrasekharan S, et al. (2007) Evaluation of anxiety, salivary cortisol and melatonin secretion following reflexology treatment: a pilot study in healthy individuals. Complement Ther Clin Pract 13: 137145. [Crossref]

6. Philippine Spa Massage Workshop. The 1st Familiarization Workshop and Boot Camp on Indigenous Therapies. (2012, January 1). Retrieved December 12, 2014

7. Fitzgerald W, Bowers E (1917) Zone therapy, or, Relieving pain at home. Columbus, Ohio: I.W. Long.

8. Kaur J, Kaur S, Bhardwaj N (2012) Effect of foot massage and reflexology on physiological parameters of critically ill patients. Nursing and Midwifery Research Journal 8: 3.

9. Moyle W, Cooke M, O'Dwyer ST, Murfield J, Johnston A, et al. (2013) The effect of foot massage on long-term care staff working with older people with dementia: a pilot, parallel group, randomized controlled trial. BMC Nurs 12: 5. [Crossref]

10. Field T, -Reif M, Hart S, Theakston H, Schanberg S, et al. (1998) Pregnant women benefit from massage therapy. J Psychosom Obstet Gynaecol 20: 31-38. [Crossref]

11. Bost N, Wallis M (2006) The effectiveness of a 15 minute weekly massage in reducing physical and psychological stress in nurses. Aust J Adv Nurs 23: 28-33. [Crossref]

12. Moeini M, Kahangi L, Valiani M, Heshmat R (2011) The effect of reflexotherapy on some of the patients' vital signs before CABG surgery. Iranian Journal of Nursing and Midwifery Research 16: 24-28.

13. Ahamadi M, Raygani AAV, Rezaee M, Heydarpour B, Taghizaeh P (2013) Comparing the effect of metatarsus and ankle reflexology massage on patients' state anxiety after coronary artery bypass graft surgery. Iran J Crit Care Nurs 6: 235-240.
14. Elshamy K, Elsafety E (2011) Effect of nursing interventions using foot reflexology on blood pressure and quality of life of hypertensive patients at' Mansoura University Hospitals: Preliminary results. Med J Cairo Univ, 79: 193-202.

15. O’Brien E, Atkins N, Stergiou G, Karpettas N, Parati G (2010) European Society of Hypertension International Protocol revision 2010 for the validation of blood pressure measuring devices in adults. Blood Press Monit 15: 23-38. [Crossref]

16. National Institute of Health and Clinical Excellence (NICE). Hypertension: clinical management of primary hypertension in adults. London: NICE; 2011. NICE clinical guideline 127.

17. Kuhn MA (1999) Complementary therapies for health care providers. Philadelphia Lippincott Williams \& Wilkins 325-326.

18. MahmoudiradGh, GhaediMoslo M, Bahrami HR (2014) Effect of foot reflexology on anxiety of patients undergoing coronary angiography. Iran J Crit Care Nurs 6: 241248.

19. Quattrin R, Zanini A, Buchini S, Turello D, Annunziata MA, et al. (2006) Use of reflexology foot massage to reduce anxiety in hospitalized cancer patients in chemotherapy treatment: methodology and outcomes. J Nurs Manag 14: 96-105. [Crossref]

20. Stephenson NL, Swanson M, Dalton J, Keefe FJ, Engelke M (2007) Partner-delivered reflexology: effects on cancer pain and anxiety. Oncol Nurs Forum 34: 127-132. [Crossref]

21. Çinar Ş, Eşer İ, Khorshıd L (2009) The Effects of Back Massage on the Vital Signs and Anxiety Level of Elderly Staying in a Rest Home. Hacettepe University Faculty of Health Sciences Nursing Journal 16: 14-21.

22. Field T, Hernandez-Reif M, Hart S, Theakston H, Schanberg S, et al. (1999). Pregnan women benefit from massage therapy. J Psychosom Obstet Gynecol 20:31-8. [Crossref]

23. Guan L, Collet J, Yuskiv N, Skippen P, Brant R, et al. (2014) The Effect of Massage Therapy on Autonomic Activity in Critically Ill Children. Evid Based Complement Alternat Med: 656750. [Crossref]

24. Delaney JP, Leong KS, Watkins A, Brodie D (2002) The short-term effects of myofascial trigger point massage therapy on cardiac autonomic tone in healthy subjects. J Adv Nurs 37: 364-371. [Crossref]

25. Black JM, Hawks JH (2009) Medical-Surgical Nursing: Clinical Management for Positive Outcomes. 8th ed. Philadelphia: Saunders/Elsevier; Chapter: Assessment of the cardiac system; 1354

26. Melzack R, Wall P (1983) The challenge of pain (Completely rev. ed.). New York: Basic Books.

27. Jeong IS (2006) Effect of self-foot reflexology on peripheral blood circulation and peripheral neuropathy in patients with diabetes mellitus. Journal of Korean Academy of Fundamentals of Nursing 13: 225-234.

28. Frankel BSM (1997) The effect of reflexology on baroreceptor reflex sensitivity, blood pressure and sinus arrhythmia. Complementary Therapies in Medicine 5: 80-84.

Copyright: $(2015$ Ramos DG. This is an open-access article distributed under the terms of the Creative Commons Attribution License, which permits unrestricted use, distribution, and reproduction in any medium, provided the original author and source are credited. 